\title{
Colour dependence of the distribution of IRAS galaxies revealed by multifractal measures
}

\author{
J. Pan \\ 1 Purple Mountain Observatory, Chinese Academy of Sciences, Nanjing 210008, PR China \\ 2 School of Physics \& Astronomy, Nottingham University, Nottingham NG7 2RD, UK \\ e-mail: Jun.Pan@nottingham.ac.uk
}

Received 6 December 2005 / Accepted 6 May 2006

\section{ABSTRACT}

\begin{abstract}
Multifractal measures are applied to three IRAS galaxy subsamples selected by colour from the PSCz catalogue. As shown by a generalised dimension spectrum, hot IRAS galaxies are found to be less clustered than cold galaxies, but the difference is very small. An alternative tool, the conditional multifractal dimension spectrum reveals an apparent peculiarity of the distribution of hot galaxies, especially at high orders. The conditional multifractal measure basically measures the environment of selected galaxies. A detailed analysis of the distribution of galaxies with their number of neighbours shows that cold galaxies are more likely to be in over-dense regions than hot galaxies. Further studies suggest that since the colour of IRAS galaxies is a good trace of star formation rate, it is possible that we have statistical evidence here for enhanced star formation rate due to galaxy interactions.
\end{abstract}

Key words. cosmology: large-scale structure of Universe - infrared: galaxies - methods: statistical

\section{Introduction}

Galaxies are inevitably affected by their environment. It has been found that early-type galaxies are far more common than spirals in centres of rich clusters, while the opposite is true in other parts of the universe (Dressler 1980; Caon \& Einasto 1995; Dressler et al. 1997). There is the same phenomenon in galaxies' spatial distribution: early-type galaxies are more strongly clustered than late-types (e.g., Hermit et al. 1996; Shepherd et al. 2001; Madgwick et al. 2003), and high luminosity galaxies are more strongly clustered than low luminosity ones (e.g., Hermit et al. 1996; Lin et al. 1996; Guzzo et al. 2000; Norberg et al. 2001). It is clear that galaxies are biased tracers of the mass, which has invoked the concept of bias to make connections between statistics of galaxies and the mass (e.g., Kaiser 1984; Bardeen et al. 1986; Mo \& White 1996; Dekel \& Lahav 1999). Galaxy samples selected by different criteria are frequently studied to quantify the phenomenon (e.g., Norberg et al. 2002; Zehavi et al. 2005).

Among the many galaxy surveys, the IRAS galaxy sample plays an important role in studying cosmic large-scale structure and galaxies' formation and evolution. Observationally, it affords large sky coverage, uniform flux calibration, good position accuracy, and insignificant galactic absorption. IRAS galaxies usually are good objects of strong star formation activities. The majority of galaxies observed in the infrared band are spirals, which tend to avoid rich galaxy clusters, so in general the infrared selected galaxy samples have a lower clustering amplitude.

The infrared emission from galaxies are produced by their gas (dust) component, illuminated mainly by UV emission from stars. Rowan-Robinson \& Crawford (1989) decomposed the spectrum of IRAS galaxies into three components: (1) a cool disk component emission from interstellar dust activated by galaxy starlight; (2) a warmer "starburst" component interpreted as a burst of star formation in the galaxy nucleus, whose spectrum is well fitted by a model of hot stars embedded in optically thick dust cloud; and (3) a "Seyfert" component that originates in a power-law continuum source within a dust cloud related to the narrow-line region of the compact source. The peak of the disk component's radiation is at the $100 \mu \mathrm{m}$ and the "starburst" part reaches maximum at $60 \mu \mathrm{m}$, while the "Seyfert" component's peaks are at $12 \mu \mathrm{m}$ and $25 \mu \mathrm{m}$; thus, the far-infrared radiation from the IRAS galaxies is a composition of ambient cirrus emission and localised emission from regions of active star formation. The relative contribution of the two components may be quantified by the dust temperature inferred from the flux ratio $S_{100} / S_{60}$.

Therefore, $S_{100} / S_{60}$, or equivalently the dust temperature, is a good measure of the star formation rate (SFR) of IRAS galaxies (Saunders et al. 1990; Mann et al. 1996). There are arguments that in deep gravity potential wells, SFRs of galaxies are effectively suppressed (e.g., Young et al. 1996; Balogh et al. 1998; Blanton et al. 1999); naturally, the segregation phenomenon in clustering is expected in the temperature or the SFR selected IRAS galaxy subsamples, which is supported by the observation that late-type optical galaxies with higher SFRs have smaller clustering amplitudes than early-type galaxies.

Surprisingly, Mann et al. (1996) found an unusual clustering scenario of their "warm" and "cold" subsamples of QDOT divided by the $36 \mathrm{~K}$ temperature criteria. Their "warm" galaxies are more strongly clustered than the "cool" galaxies, which is opposite to what one would expect. However, Hawkins et al. (2001) split the PSCz catalogue, which is superior to QDOT, into three subsamples, "hot", "warm", and "cold", which fall into temperature ranges $>31 \mathrm{~K}, 28 \mathrm{~K} \sim 31 \mathrm{~K}$, and $<28 \mathrm{~K}$, respectively, and they found that the hot galaxies are actually clustered less strongly than those cold galaxies on scales between $1 h^{-1} \mathrm{Mpc}$ and $10 h^{-1} \mathrm{Mpc}$, though the trend is weak.

Here, the problem of the colour dependence of the IRAS galaxies' clustering is revisited using a multifractal tool. For 
many years, fractal dimensions were used to test if the distribution of galaxies in the universe is homogeneous. Actually, it could have more applications in modern statistical cosmology than simply as a challenge to the Cosmological Principle. The benefit of using multifractal is that we have a whole spectrum of dimensions to describe the distribution. Actually, in the past, the fractal was already used to study morphology segregation (Wen et al. 1989; Domínguez-Tenreiro \& Martínez 1989; Domínguez-Tenreiro et al. 1994; Best et al. 1996). In addition to conventional fractal dimensions, a new tool, conditional dimension, was designed to measure differences between two samples. The idea of conditional dimensions was inspired by concepts of conditional and relative multifractal spectra (Richards \& Scheuring 1997). Details of the subsamples' construction are given in Sect. 2. In Sect. 3 we present results from ordinary multifractal measurement; Sect. 4 is dedicated to new statistics, the conditional multifractal measure. Conclusions and discussions are in the last section.

\section{Sample construction}

\subsection{The main sample from $P S C z$}

The PSCz catalogue contains 15411 galaxies covering $84 \%$ of the sky with $60 \mu \mathrm{m}$ flux, $S_{60}>0.60$ (Saunders et al. 2000); redshifts are available for 14677 galaxies with a redshift median of $\sim 0.028$. Those galaxies with galactic latitude $b<10^{\circ}$ are thrown away to exclude the high galactic absorption plane. Further selection criteria are applied by the galaxy's co-moving distance calculated with $H_{0}=100 h^{-1} \mathrm{~km} \mathrm{~s}^{-1}$ and $\Omega=1$, which is $10 h^{-1} \mathrm{Mpc}<r<250 h^{-1} \mathrm{Mpc}$ to avoid the Galaxy's local influence and make the sample in the remote end not too sparse. The final main sample for this work has 11463 galaxies.

We find that the colour of galaxies shows an apparent radial gradient. It is known that IRAS galaxies experience rapid luminosity evolution with redshift; we need to check if the colour radial gradient is an evolution effect. At $60 \mu \mathrm{m}$, Saunders et al. (1990) gives $L_{60} \propto(1+z)^{3 \pm 1}$. Although the exact luminosity evolution at $100 \mu \mathrm{m}$ is not clear, we can use the luminosity evolution at $90 \mu \mathrm{m}$, which is $L_{90} \propto(1+z)^{3.4 \pm 1}$ (Serjeant et al. 2004), as an approximation, so the colour is roughly proportional to $(1+z)^{0.4}$. The largest redshift of the sample is 0.089 which introduces correction to the colour by a factor of $\sim 1.035$ for the farthest galaxies. Even by this factor, since the mean colour of those remote galaxies is $\sim 1.1$ of our sample, the modification to the colour is about 0.04 , which is relatively small. In fact, after applying such correction to the colour, changes to the straight line fitting the $\log \left\langle S_{100} / S_{60}\right\rangle-\log r$ relation are negligible. The colour undergoes very weak evolution in the redshift regime of the sample in analysis here.

Because the main sample is flux-limited, those faint galaxies do not enter the list, and the mean luminosity will obviously increase with distance. Actually, there is a correlation between luminosity and colour: less luminous galaxies are likely to have higher colours. Therefore, those galaxies with high colour are unlikely to be selected in our main sample. The mean colour is low in places at a large distance, while the radial gradient of colour of the main sample is a selection effect. Mann et al. (1996) applied a similar mean colour-distance relation to the construction of subsamples from QDOT because they thought such a radial gradient of temperature (colour) reflects the intrinsic evolution. They found that after this correction, the clustering difference between "warm" and "cool" subsamples disappears. Now it is clear that their procedure of correction actually makes

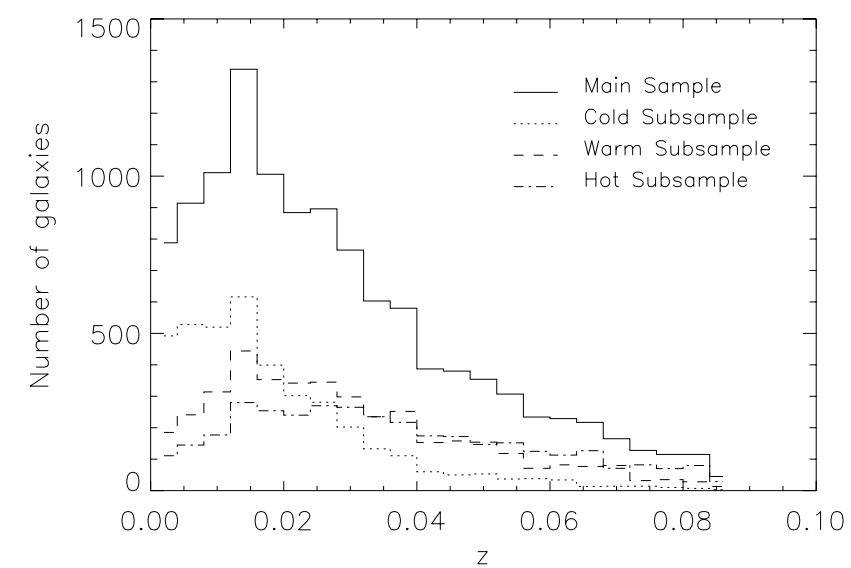

Fig. 1. Redshift distributions of galaxies of the three subsamples.

the subsamples quite contaminated: the nearby galaxies of high $S_{100} / S_{60}$ are likely to be classified as "warm", while those remote galaxies of low colour are marked as "cool".

\subsection{Colour subsamples}

Colour subsamples are generated using the method of Hawkins et al. (2001). "Cold" galaxies are defined as $S_{100} / S_{60} \geq 2.3$, the "warm" subsample consists of those of $1.7 \leq S_{100} / S_{60}<2.3$, and the hot galaxies are the remaining galaxies with $S_{100} / S_{60}<$ 1.7. Finally, there are 3917, 4010, and 3536 galaxies in the "cold", "warm" and "hot" subsamples, respectively. Differences in numbers with Hawkins et al. (2001) are due to our distance cuts. The redshift distributions of galaxies of these colour subsamples are in Fig. 1.

To estimate the error bars, we also created 20 bootstrap subsamples for each colour subsample, as well as the main sample. Scatters at the $1-\sigma$ level over the 20 bootstrap subsamples are taken as our errors.

\section{Multifractal analysis}

\subsection{The statistics and selection function}

The multifractal measure in use is constructed from the partition function

$Z(q, r)=\frac{1}{N} \sum_{i=1}^{N} p_{i}(r)^{q-1} \propto r^{\tau(q)}$,

with $p(i)=n_{i}(r) / N$, where $n_{i}(r)$ is the count of objects in the cell of radius $r$, centred upon an object labelled by $i$ (not included in the count), after corrections for the boundary effect and selection function $\psi(r)(\psi=1$ for volume-limited sample),

$n_{i}(r)=\frac{1}{f_{i}(r)} \sum_{j=1}^{N} \frac{\Psi\left(\left|\boldsymbol{r}_{j}-\boldsymbol{r}_{i}\right|-r\right)}{\psi\left(r_{j}\right)}$

where

$\Psi(x)= \begin{cases}1, & x \leq 0 \\ 0, & x>0\end{cases}$

and $f_{i}(r)$ is the volume fraction of the sphere centred on the object of radius $r$ within the boundaries of the sample. From previous works, we know that on scales larger than about $20 \sim 30 h^{-1} \mathrm{Mpc}$, the distributions of galaxies are homogeneous 


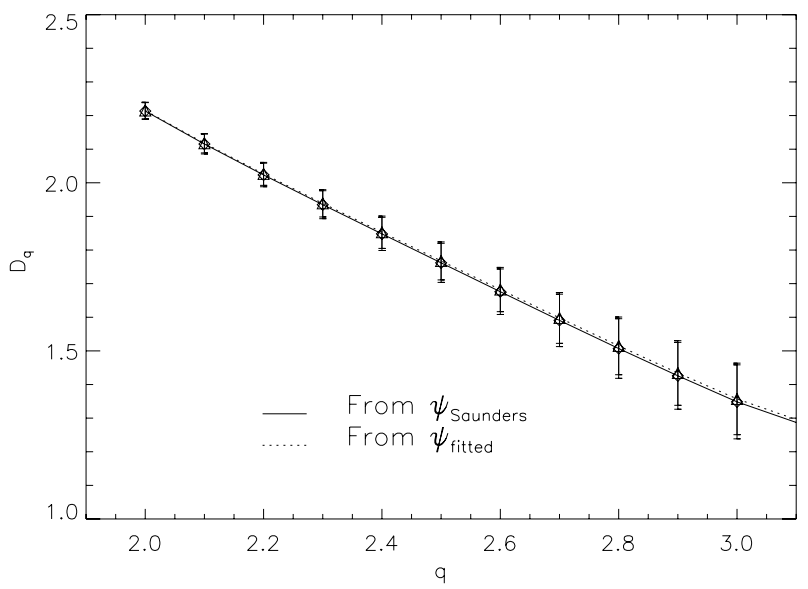

Fig. 2. Dimension spectrum of $q \in[2,3]$ of the main sample under corrections of different selection functions. One is from Saunders et al. (2000) and the other is acquired from a direct numerical fit of $N-z$. The generalised dimensions are fitted at scales $r<10 h^{-1} \mathrm{Mpc}$.

(Pan \& Coles 2000); the comparison here focuses on scales less than $10 h^{-1} \mathrm{Mpc}$ to ensure reliable scaling features. Since the boundary correction does not significantly modify the result for the PSCz sample, especially on small scales (Pan \& Coles 2002), to reduce the computation burden, the volume correction method is adopted to deal with boundaries. $\tau(q)$ by

The Rényi dimension $D_{q}$ is derived from the mass exponent

$D_{q}=\tau(q) /(q-1)$

When $q=2$, the partition function $Z$ is the correlation integral and $D_{2}$ is the correlation dimension. For each value of $q, Z(q)$ and $D_{q}$ give information about the scaling properties of different aspects of the density field. For high $q$, they are dominated by high-density regions, while for low $q$ and $q<0$ the measure is weighted toward low-density regions. Note for $q=1$, the partition function is defined differently as $Z(q=1, r)=\sum_{i} \log p(i) \sim$ $r^{D_{1}}$ (cf. Martínez \& Saar 2002). In this paper, we concentrate on the moments of order $q \geq 2$.

From Fig. 1 we know that the redshift distributions of the galaxies of the subsamples are not the same. Selection functions of subsamples have to be estimated separately. Of course, it is optimal to use a maximum-likelihood method to generate selection functions for all subsamples. Here we just simply smooth those curves and then fit the distance-density relation via a nonlinear least-square fitting to a double power-law function, as in Saunders et al. (2000),

$\psi(r)=\psi_{*}\left(\frac{r}{r_{*}}\right)^{1-\alpha}\left[1+\left(\frac{r}{r_{*}}\right)^{\gamma}\right]^{-(\beta / \gamma)}$,

in which $\psi_{*}, \alpha, r_{*}, \gamma$, and $\beta$ are fitting parameters. A comparison of the partition functions of the main sample with the selection function from Saunders et al. (2000) indicates the accuracy of this "naive" method and that our simple fits are good enough for the statistics (see Fig. 2). So, for all colour subsamples, we adopt the nonlinear least-square fitted selection functions.

\subsection{Results of multifractal analysis}

Measurements of $Z(q, r)$ at different orders of our colour subsamples are displayed in Fig. 3; error bars are estimated from
20 bootstrap subsamples for each colour subsample. It is obvious that on large scales the distributions of all subsamples become homogeneous, which means the generalised dimensions are all close to 3 . The scales of interests are therefore below $\sim 20 h^{-1} \mathrm{Mpc}$, a level at which the distribution is far from homogeneity. On the other hand, to avoid severe discreteness effects, measurements on scales of less than $1 h^{-1} \mathrm{Mpc}$ are also cut off. In the regime of $\sim 1-10 h^{-1} \mathrm{Mpc}$, local dimensions are approximately constant with small fluctuation, and the generalised dimensions $D_{q}$ are calculated by fitting the partition function in this scale range. Note that $Z(q, r)$, on small scales of all the colour subsamples, appears irregular with large error bars when $q>3$, so one should be careful to use the dimensionality obtained by a regression of $\log Z(q>3)-\log r$.

Comparisons of the $D_{q}$ of the subsamples are displayed in Fig. 4. The most important dimension is the correlation dimension $D_{2}$. For the "hot", "warm", and "cold" subsamples, $D_{2}$ is $2.34 \pm 0.07,2.16 \pm 0.06$, and $2.14 \pm 0.03$, respectively. Therefore, cold galaxies are distributed with a smaller fractal dimension than hot galaxies, which tells us that cold galaxies are more strongly clustered.

The dimensionality differences among the three subsamples become smaller for higher order moments. High order moments are dominated by those cells from regions with large number of galaxies, while contributions from cells in sparse regions is effectively suppressed. So, it is possible that the richest regions of all the subsample obey nearly the same scaling law. Unfortunately, the method we used here is unable to estimate the other half of the dimension spectrum of $q<2$, otherwise, it is probable that we would find bigger differences. Of course, due to large uncertainties, one should be conservative about this observation.

Although the partition functions of all three subsamples seem to asymptotically agree with each other on large scales within error bars, their selection functions and corresponding normalisation factors are different, so we cannot compare the amplitudes of the partition functions directly.

\section{Conditional multifractal measure}

\subsection{Definition}

It is a tradition to split a main sample into several subsamples to explore differences in their spatial distributions. This treatment wastes information provided by the configuration of relative positions among galaxies of different subsamples. For many years the only tool to overcome this was the cross-correlation function. The mark correlation functions, an elegant measure recently introduced into statistical cosmology are ideal for quantifying the morphology dependence of clustering (Beisbart \& Kerscher 2000; Szapudi et al. 2000; Sheth 2005). Using the mark correlation functions it has already been found that there is no luminosity dependence of clustering in the PSCz catalogue (Szapudi et al. 2000).

The environment of galaxies of a particular type is a concept closely related to cross-correlation. The partition sum of Eq. (1) measures moment of the counts of neighbours; it is naturally a good tool to study the environment, if we include galaxies of all types as neighbours instead of only galaxies of the same type as the centre galaxy. The new measure is given a name of "conditional multifractal" (Richards \& Scheuring 1997). In the following, we give its formal definition.

Let the main sample of $N$ points be $\mathcal{S}=\cup_{m=1}^{M} S_{m}$, of which $S_{m=1, \ldots, M}$ is a subsample in which there are $N_{m}^{m=1}$ objects marked 

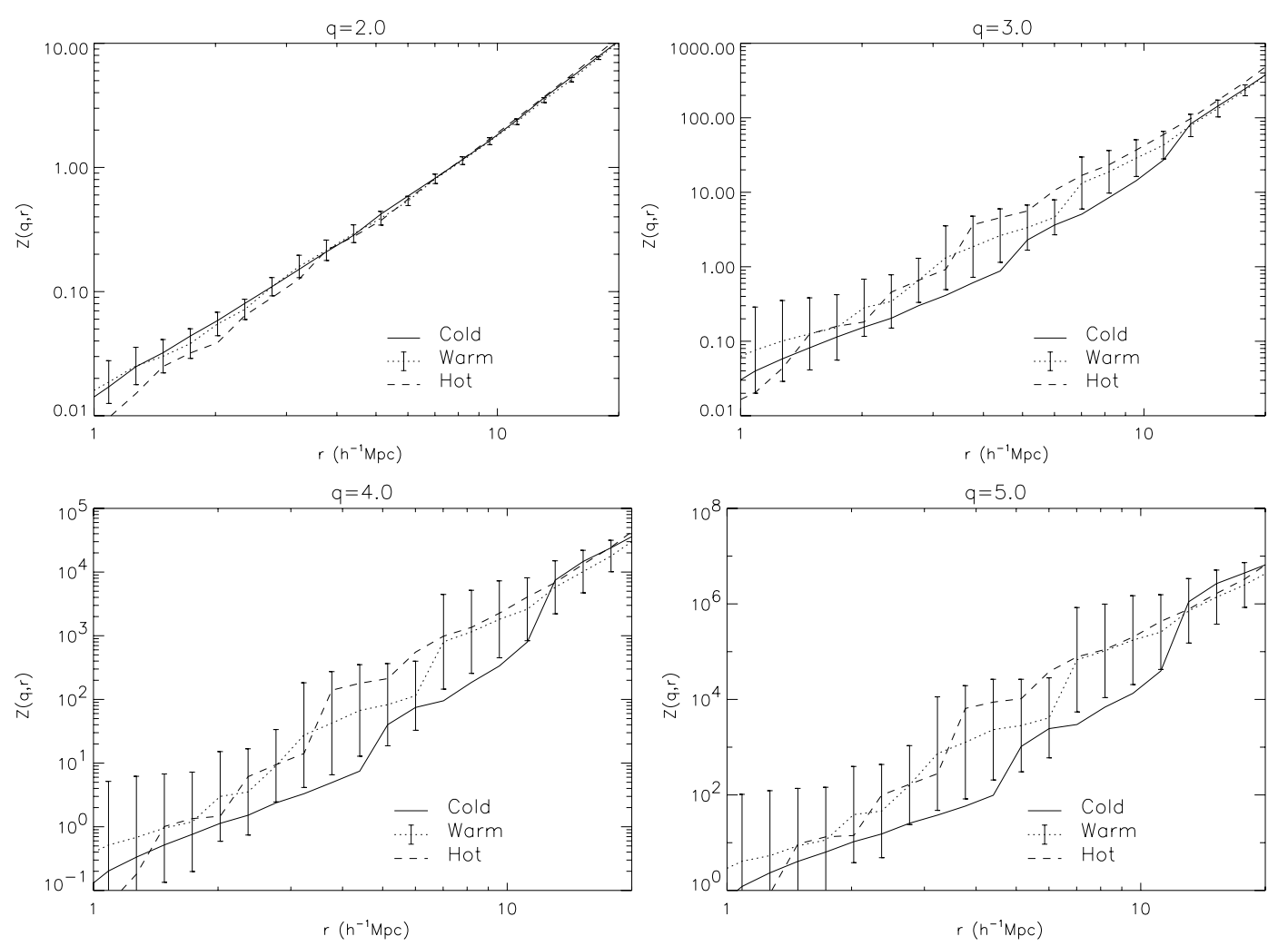

Fig. 3. $Z(q, r)$ of $q=2,3,4$, and 5 for colour subsamples. Only error bars of the warm subsample are plotted; the other two subsamples have approximately the same size error bars.

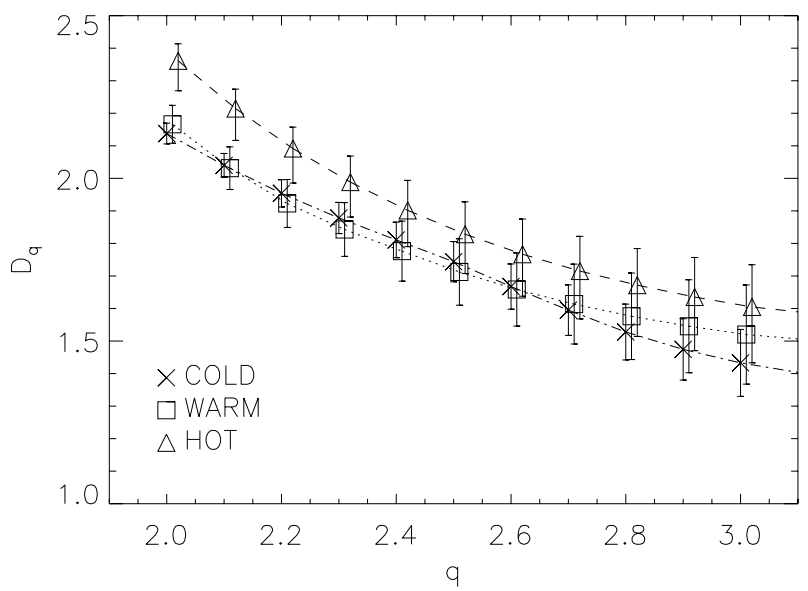

Fig. 4. Generalised dimensions $D_{q}$ of $q \in[2,3]$ in $r \in\left(1.5,10 h^{-1} \mathrm{Mpc}\right)$ for colour subsamples; points of the "warm" and the "hot" subsamples are slightly shifted to the right for better presentation.

by their position vectors $\boldsymbol{r}_{m_{i}}$ and $\left\{\boldsymbol{r}_{j} \mid j=1, \ldots, N\right\}=\cup_{m=1}^{M}\left\{\boldsymbol{r}_{m_{i}} \mid i=\right.$ $\left.1, \ldots, N_{m}\right\}$. The conditional partition function of $S_{m}$ is thus

$Z_{c}^{(m)}(q, r)=\frac{1}{N_{m}} \sum_{i=1}^{N_{m}}\left[\frac{\sum_{j=1, j \neq m_{i}}^{N} \Psi\left(\left|\boldsymbol{r}_{j}-\boldsymbol{r}_{m_{i}}\right|-r\right)}{N}\right]^{q-1}$.

For our flux limited sample, we need to apply the same corrections to the kernel that we did in Eq. (2).

Formally, by writing the measure of neighbours in the $j$ th cell as $\mu_{j}, j=1, \ldots, N$, the conditional mass exponent $\tau_{\mathrm{c}}$ and the spectrum of the generalised conditional dimension $D_{\mathrm{c}}(q)$ of the subsample $S_{m}$ is

$\tau_{\mathrm{c}}^{(m)}(q) \equiv \frac{\mathrm{d} \log Z_{\mathrm{c}}^{(m)}}{\mathrm{d} \log r}=\frac{\mathrm{d} \log \sum_{i=1}^{N_{m}} \mu_{m_{i}}^{q-1}}{\mathrm{~d} \log r}, D_{\mathrm{c}}^{(m)}(q) \equiv \frac{\tau_{\mathrm{c}}^{(m)}}{q-1}$.

When $q=2, Z_{\mathrm{c}}(q=2, r)$ is the conditional correlation integral and $D_{\mathrm{c}}(2)$ is the conditional correlation dimension.

The subset is a collection of specially picked sampling points of the main set, since

$\sum_{j=1}^{N} \mu_{j}^{q-1}=\sum_{m=1}^{M} \sum_{i=1}^{N_{m}} \mu_{m_{i}}^{q-1}$

the relation between $D_{q}$ and $D_{\mathrm{c}}(q)$ is

$D_{q}=\sum_{m=1}^{M}\left[\frac{\sum_{i=1}^{N_{m}} \mu_{m_{i}}^{q-1}}{\sum_{j=1}^{N} \mu_{j}^{q-1}} \times D_{\mathrm{c}}^{(m)}(q)\right]$.

If a subsample $S_{m}$ is an uniform dilution of $\mathcal{S}$, $\sum_{i=1}^{N_{m}} \mu_{m_{i}}^{q-1} / \sum_{j=1}^{N} \mu_{j}^{q-1}=N_{m} / N$ and $D_{\mathrm{c}}^{(m)}(q)=D_{q}$. Any deviation from such uniformity is embedded in the conditional dimension; the conditional multifractal measure is therefore an effective tracer of differences among subsamples.

\subsection{Results of conditional multifractal analysis}

Conditional partition functions and conditional generalised dimensions are calculated for each colour subsample, and results are displayed in Figs. 5 and 6. Recall that neighbours of an object are those in the main sample, so only the selection function provided by Saunders et al. (2000) is needed. The error bars are from 20 bootstrap subsamples. 

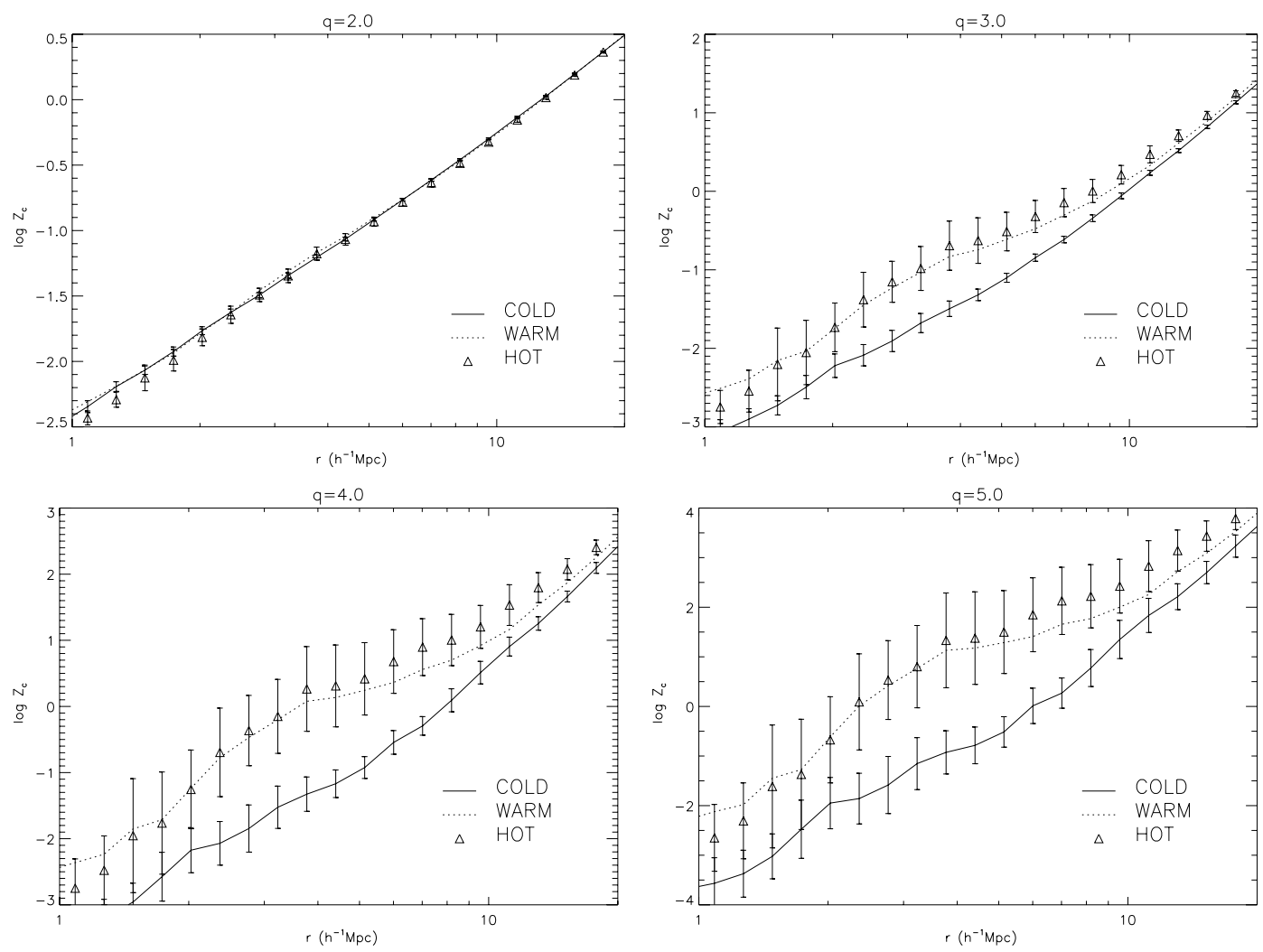

Fig. 5. $Z_{\mathrm{c}}(q, r)$ of $q=2,3,4$, and 5 of the PSCz colour subsamples. Error bars of the "warm" subsample are not plotted; they are very similar in size to those of the "hot" subsample.

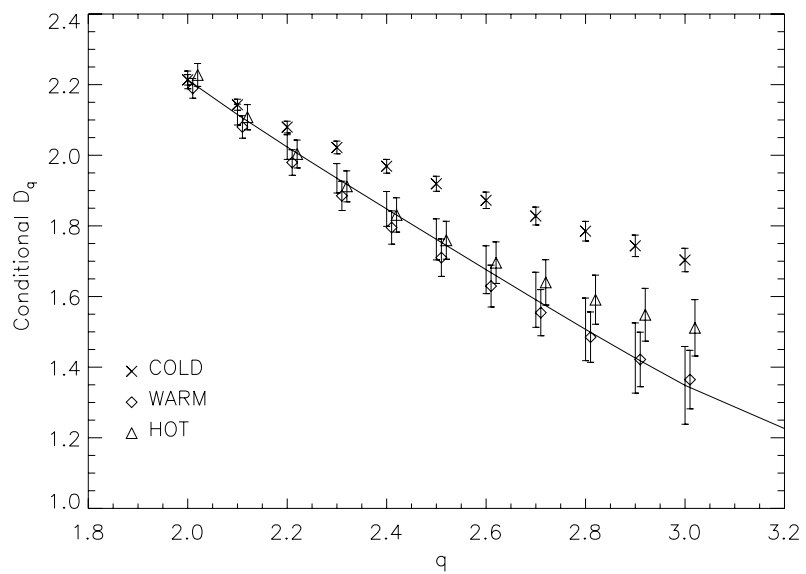

Fig. 6. The conditional dimension spectrum within $q \in[2,3]$ and $r \in(1,10) h^{-1} \mathrm{Mpc}$. Points of the "warm" and the "hot" subsamples are slightly shifted to the right. The solid line with error bars is the dimension spectrum of the main sample.

Contrary to the ordinary multifractal measure, the conditional dimensions $D_{\mathrm{c}}(2)$ of the colour subsamples are consistent with each other within error bars, while higher orders differ. We can see that conditional dimension spectrum of the "hot" and the "warm" subsamples are in good agreement with the spectrum of the main sample within error bars, which means the two subsamples are basically uniformly diluted realisations of the main sample. The "cold" subsample has a quite different dimension spectrum; its conditional dimensions are larger than those of the "hot" subsample at high orders, e.g., for $q=3, D_{\mathrm{c}}^{\text {(cold) }}=1.70 \pm 0.04$, and $D_{\mathrm{c}}^{\text {(hot) }}=1.50 \pm 0.08$. One thing that needs to be addressed is that while the conditional dimensions are a measure of the environment of the central object, a larger conditional dimension does not mean that the environment is less clustered, it just tells us that the class of objects that are measured are in regions of a special scaling property or "strangeness", in fractal language.

On large scales, the distribution of galaxies is asymptotically homogeneous, and the $Z_{\mathrm{c}}(q, r)$ of different subsamples are consistent within error bars (Fig. 5). So, if the averaged environment of subsamples on small scales is different, it will be shown by the amplitudes of $Z_{\mathrm{c}}(q, r)$. For $q=2, Z_{\mathrm{c}}$, the mean number of neighbours, of all the colour subsamples agree with each other within error bars. When $q>2, Z_{\mathrm{c}}^{\text {(cold) }}$ is much smaller than $Z_{\mathrm{c}}^{(\text {hot })}$. Since $\langle\mu\rangle_{\text {cold }}=\langle\mu\rangle_{\text {hot }}$, the variance in the number of neighbours around cold galaxies $\left\langle(\mu-\langle\mu\rangle)^{2}\right\rangle_{\text {cold }}=Z_{c}^{\text {(cold) }}(q=3)-Z_{c}^{\text {(cold)2 }}(q=2)$ is smaller than that around the hot and the warm galaxies, hence the skewness.

At high $q, Z_{\mathrm{c}}(q, r)$ has similar properties to the ordinary $Z(q, r)$ and is also weighted toward rich cells. It is interesting to see the distribution of richness around galaxies. As an example, the distribution function of the number of neighbours around galaxies of colour subsamples at scale $r \sim 4 h^{-1}$ Mpc are shown in Fig. 7. Clearly, hot and warm galaxies have higher possibilities of being located in very sparse regions than cold galaxies, and cold galaxies are more likely distributed in rich environments. Cases at other scales less than $10 h^{-1} \mathrm{Mpc}$ are similar.

\section{Discussion}

In this work, colour subsamples of PSCz galaxies are analysed with two kinds of multifractal measures; all analysis clearly denotes that their spatial distributions are different. The ordinary multifractal measure of our three colour subsamples gives results 


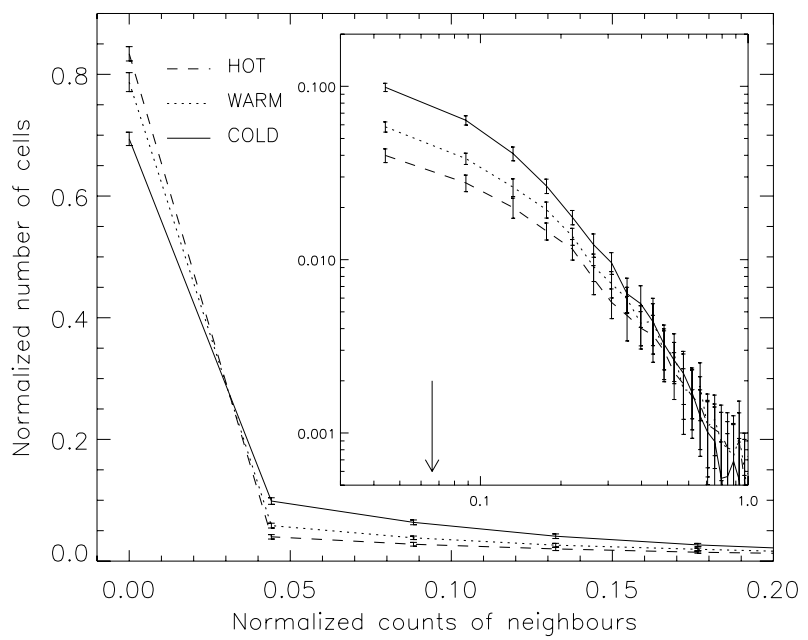

Fig. 7. Distribution of the number of neighbours of galaxies in different colour subsamples; the inset plot is the log-log version for a better demonstration, and the vertical arrow in the subplot marks the average normalised neighbour count for a randomly selected galaxy belonging to the main sample. The radius of the cell is $r=3.77 h^{-1} \mathrm{Mpc}$. Counts of neighbours are normalised by the number of all galaxies, which is 11463 , here. The number of cells is counted within a bin of width 0.044 , then it is normalised for each colour subsample by the total number of galaxies of that subsample. Error bars are estimated over 20 bootstrap subsamples.

consistent with the normal morphological segregation scenario of galaxy clustering. Hot galaxies are clustered less strongly with a larger correlation dimension $D_{2}$ than cold galaxies. The difference is not very strong, and actually, higher-order dimensions fail to detect a significant difference.

Details of the spatial distribution of galaxies of different colours are more clearly displayed by the new statistics - the conditional multifractal that is designed to measure the environment around galaxies of a particular type. The conditional correlation integrals and conditional correlation dimensions are the same for all colour subsamples within error bars; at high orders $q>2$, conditional multifractal dimensions detect marginal differences. It is found that cold galaxies are in regions with less "strangeness" than hot galaxies.

If the model of IRAS galaxies by Rowan-Robinson \& Crawford (1989) is reasonable, the colour defined in the paper is closely associated with the SFR of galaxy. Effects of environment on star formation activity are very complex under competing mechanisms. In rich environments, spirals exhibit significant gas deficiencies resulting from ram pressure from intracluster mediums, together with possible tidal stripping from interactions with other galaxies and cluster potential; in this way, star formation rate is effectively suppressed. On the other hand, there are external environmental influences that can have much stronger effects on boosting the star formation rate. Studies of $\mathrm{H} \alpha$ and FIR emission of interacting and merging galaxies have shown strong excess star formation (see review of Kennicutt 1998). Numerical simulations also pointed out pair interaction, and merging can produce tidal gas inflows during orbit decay; the inflow will drastically boost the star formation (e.g., Barnes \& Hernquist 1996; Tissera 2000; Tissera et al. 2002).

As displayed in Fig. 7, in all subsamples, the possibility of finding an isolated galaxy is much higher than having galaxies with neighbours, while the "hot" subsample has a larger portion of galaxies with no companion than the "cold" subsample. The poor possibility of finding a galaxy with prominent present star formation activities in a rich environment can be interpreted as follows: in crowded regions, most galaxies experienced an active star formation stage on a very short time scale at high redshift, and then were quenched afterwards. The galaxies that were still there with considerable present SFR are likely the small number of survivors. This conclusion is similar to the analysis of the 2dFGRS and SDSS surveys by Balogh et al. (2004). Actually, observations of Spitzer have shown that the bulk of star formation in massive galaxies occurs at early cosmic epochs and is largely complete by $z \sim 1.5$, while at $z<1$ lower-mass galaxies dominate the overall cosmic mass assembly (Papovich 2006). It is highly possible that the IRAS galaxies in rich regions are of small mass, which is consistent with the discovery of Martínez et al. (2002). Another aspect is that the field IRAS galaxies are probably systems of slow evolution, which may be tested by studies on the SFRs of galaxies with a "loose" friend and galaxies without a neighbour over a period of time.

Nevertheless, in Fig. 7, with the increasing number of neighbours, the probability of a hot galaxy living with such richness is smaller than that of a cold galaxy. This picture shows the downside effects of environment on the SFRs of galaxies, which is consistent with recent discoveries that high SFR galaxies are inhibited in rich environments in the 2dFGRS and SDSS surveys (Martínez et al. 2002; Domínguez et al. 2002; Balogh et al. 2004).

In the high richness regime, the distribution of hot and cold galaxies are asymptotically in agreement with each other within error bars. There must also be some mechanism that effectively enhances the galaxy's star formation rate, partly in compensation for the general suppression when a region becomes very crowded, since otherwise the distribution curve of the "hot" subsample should be always lower than the "cold" subsample. Krongold et al. (2002) found that interacting IRAS galaxies have lower $S_{100} / S_{60}$ than isolated galaxies. It has also been found that in very dense regions, there is induced star formation in galaxy pairs at a very small separation, due to interactions, and that a galaxy with a high star formation rate is likely to trigger more star formation in its close companion (e.g., Carter et al. 2001; Tissera et al. 2002; Lewis et al. 2002; Sol Alonso et al. 2004, 2006). However, in this regime, our distribution curves have large uncertainties as there are not many cells within the bin for counting since IRAS galaxies avoid rich clusters. We shall be conservative about this claim, which needs confirmation from future works with more complete and denser samples than the PSCz.

Acknowledgements. The author thanks the anonymous referee for very helpful comments, and appreciates useful suggestions from Peter Coles. The author also acknowledges the hospitality and kindness of the Astronomy Department of Peking University and the Beijing Astronomical Planetarium.

\section{References}

Balogh, M., Eke, V., Miller, C., et al. 2004, MNRAS, 348, 1355 Balogh, M. L., Schade, D., Morris, S. L., et al. 1998, ApJ, 504, L75 Bardeen, J. M., Bond, J. R., Kaiser, N., \& Szalay, A. S. 1986, ApJ, 304, 15

Barnes, J. E., \& Hernquist, L. 1996, ApJ, 471, 115

Beisbart, C., \& Kerscher, M. 2000, ApJ, 545, 6

Best, J. S., Charlton, J. C., \& Mayer-Kress, G. 1996, ApJ, 456, 55

Blanton, M., Cen, R., Ostriker, J. P., \& Strauss, M. A. 1999, ApJ, 522, 590

Caon, N., \& Einasto, M. 1995, MNRAS, 273, 913

Carter, B. J., Fabricant, D. G., Geller, M. J., Kurtz, M. J., \& McLean, B. 2001, ApJ, 559, 606

Dekel, A., \& Lahav, O. 1999, ApJ, 520, 24

Domínguez, M. J., Zandivarez, A. A., Martínez, H. J., et al. 2002, MNRAS, 335, 825 
Domínguez-Tenreiro, R., \& Martínez, V. J. 1989, ApJ, 339, L9

Domínguez-Tenreiro, R., Campos, A., Gomez-Flechoso, M. A., \& Yepes, G. 1994, ApJ, 424, L73

Dressler, A. 1980, ApJ, 236, 351

Dressler, A., Oemler, A. J., Couch, W. J., et al. 1997, ApJ, 490, 577

Guzzo, L., Barlett, J. G., Cappi, A., et al. 2000, A\&A, 355, 1

Hawkins, E., Maddox, S., Branchini, E., \& Saunders, W. 2001, MNRAS, 325, 589

Hermit, S., Santiago, B. X., Lahav, O., et al. 1996, MNRAS, 283, 709

Kaiser, N. 1984, ApJ, 284, L9

Kennicutt, R. C. 1998, ARA\&A, 36, 189

Krongold, Y., Dultzin-Hacyan, D., \& Marziani, P. 2002, ApJ, 572, 169

Lewis, I., Balogh, M., De Propris, R., et al. 2002, MNRAS, 334, 673

Lin, H., Kirshner, R. P., Shectman, S. A., et al. 1996, ApJ, 471, 617

Madgwick, D. S., Hawkings, E., Lahav, O., et al. 2003, MNRAS, 344, 847

Mann, R. G., Saunders, W., \& Taylor, A. N. 1996, MNRAS, 279, 636

Martínez, H. J., Zandivarez, A., Domínguez, M., Merchán, M. E., \& Lambas, D. G. 2002, MNRAS, 333, L31

Martínez, V. J., \& Saar, E. 2002, Statistics of the Galaxy Distribution (Boca Raton: Chapman \& Hall/CRC Press)

Mo, H. J., \& White, S. D. M. 1996, MNRAS, 282, 347

Norberg, P., Baugh, C. M., Hawkins, E., et al. 2001, MNRAS, 328, 64

Norberg, P., Baugh, C. M., Hawkins, E., et al. 2002, MNRAS, 332, 827

Pan, J., \& Coles, P. 2000, MNRAS, 318, L51
Pan, J., \& Coles, P. 2002, MNRAS, 330, 719

Papovich, C. 2006, New Astron. Rev., 50, 134

Richards, R. H., \& Scheuring, I. 1997, Fractals, 5, 153

Rowan-Robinson, M., \& Crawford, J. 1989, MNRAS, 238, 523

Saunders, W., Rowan-Robinson, M., Lawrence, A., et al. 1990, MNRAS, 242, 318

Saunders, W., Sutherland, W. J., Maddox, S. J., et al. 2000, MNRAS, 317, 55

Serjeant, S., Carramiñana, A., Gonzáles-Solares, E., et al. 2004, MNRAS, 355, 813

Shepherd, C. W., Carlberg, R. G., Yee, H. K. C., et al. 2001, ApJ, 560, 72

Sheth, R. K. 2005, MNRAS, 364, 796

Sol Alonso, M., Tissera, P. B., Coldwell, G., \& Lambas, D. G. 2004, MNRAS, 352,1081

Sol Alonso, M., Lambas, D. G., Tissera, P., \& Coldwell, G. 2006, MNRAS, 367, 1029

Szapudi, I., Branchini, E., Frenk, C. S., Maddox, S., \& Saunders, W. 2000, MNRAS, 318, L45

Tissera, P. B. 2000, ApJ, 534, 636

Tissera, P. B., Domínguez-Tenreiro, R., Scannapieco, C., \& Sáiz, A. 2002, MNRAS, 333, 327

Wen, Z., Deng, Z.-G., Liu, Y.-Z., \& Xia, X.-Y. 1989, A\&A, 219, 1

Young, J. S., Allen, L., Kenney, J. D. P., Lesser, A., \& Rownd, B. 1996, AJ, 112, 1903

Zehavi, I., Zheng, Z., Weinberg, D. H., et al. 2005, ApJ, 630, 1 